\title{
Crystals and vessel walls
}

\author{
P. N. PLATT AND A. MALCOLM
}

From the Departments of Rheumatology and Pathology, University of Newcastle upon Tyne

\begin{abstract}
Hitherto the concept of crystal-induced diseases has had its main impact in rheumatology. It is becoming obvious, however, that crystals have a part to play in the production of disease in other systems. We thought that the crystalline form of some of the constituents of atherosclerotic plaques may play a part in their formation-for example, cholesterol crystals are capable of activating polymorphs ${ }^{1}$ and promoting collagen production by fibroblasts. ${ }^{2}$ Hence as part of an investigation into the role of crystals in vessel walls we undertook a preliminary study using polarising light microscopy and present the results.

Early non-calcified plaques of uncomplicated atheroma of the aorta were obtained at necropsy from both sexes with an age range of 28-84 years.

These plaques were subjected to rapid freezing and $5 \mu \mathrm{m}$ thick cryostat
\end{abstract}

sections both unstained and stained (haematoxylin and eosin, oil red ' $O$ ') were examined using a polarising light microscope. The oil red ' $O$ ' demonstrated lipid, and crystals of cholesterol and monosodium urate. Urate crystals were identified by their characteristic appearance under polarised light microscopy and solubility in uricase.

In addition to the expected cholesterol crystals we describe what appeared to be crystals of monosodium urate occurring in samples of early atheromatous plaques from human aortas at necropsy. We believe that this may be of some importance in view of the long speculated association between hyperuricaemia and atherosclerosis. ${ }^{3}$ The situation may be analogous to that occurring in tophi, where cholesterol and hydroxyapatite ${ }^{4}$ are often found in conjunction with urate crystals suggesting common nucleation factors. The capabilities of these crystals to trigger inflammatory and sclerotic reactions suggests a possible role in the production of atherosclerotic lesions.

\section{References}

1 Hammerschmidt D E, Greenberg C S, Yamada O, Craddock P R, Jacob H S. Cholesterol and atheroma lipids activate complement and stimulate granulocytes. $J$ Lab Clin Med 1981; 98: 68-77.

2 Pritzker K P H, Adel G F, Omar S, Gertzbein S D. Experimental cholesterol crystal arthropathy.J Rheumatol 1981;8: 281-90.

3 Dreyfuss $F$. The role of hyperuricaemia in coronary artery disease. Diseases of the Chest 1960; 38: 332-6.

4 Dieppe P. Crystal deposition disease and the soft tissues. Clinics in Rheumatic Diseases 1979; 5: 807-21.

\section{An immunoelectron microscopical study of the orientation of $\mathrm{IgG}$ molecules on the surface of monosodium urate crystals}

\author{
T. BARDIN, ${ }^{1}$ P. V. CHERIAN, ${ }^{2}$ AND H. R. SCHUMACHER ${ }^{2}$
}

From the ${ }^{1}$ Clinique Rheumatologique, Hopital Lariboisière, Paris, France, and ${ }^{2}$ University of Pennsylvania School of Medicine, Philadelphia, USA

The binding of IgG to monosodium urate (MSU) crystals has been demonstrated by several techniques. ${ }^{1-3}$ It is thought to play an important part in the pathophysiology of the gouty attack, as IgG coating of MSU crystals could modulate crystal interactions with cells, in particular by reacting with IgG Fc receptors. The availability of Fc fragments on the crystal-bound IgGs thus appears to be an important factor in the part IgG might play in gouty inflammation. We recently presented data in favour of such an availability. ${ }^{3}$ We set out to investigate further the orientation of IgG molecules from serum, bound to MSU crystals, by using immunoperoxidase techniques directed against the $\mathrm{Fc}$ and $\mathrm{F}\left(\mathrm{ab}^{\prime}\right)_{2}$ fragments of $\mathrm{IgG}$.

Unheated synthetic MSU crystals were incubated with normal human serum for one hour at $4^{\circ} \mathrm{C}$, washed, and then processed with an indirect immunoperoxidase technique using rabbit antihuman IgG, at a concentration of $120 \mu \mathrm{g} / \mathrm{ml}$, directed either against the $F\left(a b^{\prime}\right)_{2}$ or the $F c$ fragments, followed by horseradish peroxidase conjugated goat antirabbit IgG. Next, crystals were incubated with $\mathrm{DAB}+\mathrm{H}_{2} \mathrm{O}_{2}$ and were post fixed 